Draft Version March 3, 2022

Typeset using LATEX twocolumn style in AASTeX61

\title{
DETECTION OF GEV GAMMA-RAYS FROM HESS J1534-571 AND MULTIWAVELENGTH IMPLICATIONS FOR THE ORIGIN OF THE NON-THERMAL EMISSION
}

\author{
Miguel Araya ${ }^{1}$
}

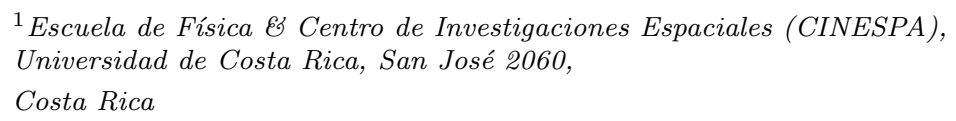

\section{ABSTRACT}

HESS J1534-571 is a very high energy gamma-ray source that was discovered by the H.E.S.S. observatory and reported as one of several new sources with a shell-like morphology at $\mathrm{TeV}$ energies, matching in size and location with the supernova remnant G323.7-1.0 discovered in radio observations by the Molonglo Galactic Plane Survey. Many known TeV shells also show X-ray emission, however, no X-ray counterpart has been seen for HESS J1534-571. The detection of a new $\mathrm{GeV}$ source using data from the Fermi satellite that is compatible in extension with the radio supernova remnant and shows a very hard power-law spectrum $\left(\frac{d N}{d E} \propto E^{-1.35}\right)$ is presented here, together with the first broadband modeling of the nonthermal emission from this source. It is shown that leptonic emission is compatible with the known multiwavelength data and a corresponding set of physical source parameters is given. The required total energy budget in leptons is reasonable, $\sim 1.5 \times 10^{48} \mathrm{erg}$ for a distance to the object of $5 \mathrm{kpc}$. The new GeV observations imply that a hadronic scenario, on the other hand, requires a cosmic ray spectrum that deviates considerably from theoretical expectations of particle acceleration.

Keywords: gamma rays: ISM — ISM: individual (HESS J1534-571) — ISM: supernova remnants 


\section{INTRODUCTION}

Supernova remnants (SNRs) have been considered the main sources of Galactic cosmic rays for a long time from energetic considerations. Charged particles are thought to gain energy by the mechanism of diffusive shock acceleration (e.g., Krymskii 1977; Bell 1978) in the blast waves of supernova explosions. The process of cosmic ray scattering is thought to be enhanced by the presence of magnetic field fluctuations (e.g., Bell 1978, 2004) that could allow some particles to reach $\mathrm{PeV}$ energies and still be able to remain confined within the sources. Observations of several SNRs at $\mathrm{MeV}-\mathrm{GeV}$ energies with the Fermi Large Area Telescope (LAT, Atwood et al. 2009) have revealed the characteristic gamma-ray spectrum expected from the decay of neutral pions produced in hadronic interactions in several sources located near or interacting with high-density material (Ackermann et al. 2013; Jogler \& Funk 2016). These sources are middle-aged $\left(\sim 10^{4} \mathrm{yr}\right)$ and show a break in the spectrum at $\mathrm{GeV}$ energies.

Other types of SNRs that produce gamma-rays are TeV shells such as RX J1713.7-3946, RCW 86 and RX J0852.0-4622, which show hard power-law GeV spectra of the form $E^{-\Gamma}$, with $\Gamma \sim 1.5$ and 1.4 in the first two cases. Such a spectral shape can naturally be explained by interactions of high-energy leptons with ambient photon fields through the process of inverse Compton scattering (IC) (e.g., Acero et al. 2015a). For other types of SNRs, mixed contributions from hadronic emission and leptonic processes (including perhaps considerable nonthermal bremsstrahlung) are possible (e.g., Araya \& Cui 2010).

For the synchrotron-dominated TeV shell RX J1713.73946 , which shows very low thermal X-ray emission and thus low average gas densities, it has been proposed that hadronic processes could account for the hard gammaray spectrum when very energetic cosmic rays penetrate dense and cold clumps of matter located within the shell (see, e.g., Aharonian \& Atoyan 1996; Fukui et al. 2012; Gabici \& Aharonian 2014; The Fermi-LAT Collaboration 2017). In order to identify the nature of the gammarays from SNRs constraining the values of ambient parameters and properties is crucial, and this can be done through multiwavelength observations.

HESS J1534-571 was discovered at TeV energies by the High Energy Stereoscopic System (H.E.S.S.), an imaging atmospheric Cherenkov telescope (Pühlhofer et al. 2015). It was classified as a TeV-SNR of the shell type from its morphology. The associated SNR candidate, G323.7-1.0, was also recently discovered in radio observations of the Molonglo Galactic Plane Survey, which revealed an extremely faint $\sim 0.9^{\circ} \times 0.6^{\circ}$ oval shell (Green et al. 2014) that matches the TeV emission. Pühlhofer et al. (2015) note that no X-ray emission in the region of HESS J1534-571 has been detected from the ROSAT survey data, and this could be explained from Galactic absorption. However they also note that no X-ray emission has been seen in several Suzaku observations that covered parts of the shell. The absence of X-ray synchrotron emission would make HESS J1534-571 atypical as other TeV shells such as RX J1713.7-3946, SN 1006, RCW 86, RX J0852.0-4622 and HESS J1731-347, show non-thermal X-ray emission (e.g., Takeda et al. 2016; Ajello et al. 2016; Araya \& Frutos 2012; Capasso et al. 2016; Sano et al. 2015). There are, however, $\mathrm{TeV}$ shell candidates with no known X-ray counterpart such as HESS J1614-518 (Sakai et al. 2011) and HESS J1912+101 (Pühlhofer et al. 2015).

In this work, the detection of an extended $\mathrm{GeV}$ source with the LAT at the location of the SNR G323.7-1.0 is shown. No previous source has been reported at this location in the LAT Third Source Catalog, the latest catalogs of hard LAT sources nor the First Fermi LAT Supernova Remnant Catalog (Acero et al. 2015b; Ackermann et al. 2016; Acero et al. 2016; The Fermi-LAT Collaboration 2017; The Fermi LAT Collaboration et al. 2017). The source extension matches the radio shell and it shows a hard $\mathrm{GeV}$ spectrum. The combined gammaray observations from the Fermi LAT satellite and preliminary H.E.S.S. fluxes can be explained within the framework of a simple one-zone leptonic model and a set of possible source parameter values is derived. Finally, a hadronic scenario for the $\mathrm{GeV}-\mathrm{TeV}$ emission is also studied, although this scenario is less likely since the required particle spectrum deviates considerably from standard predictions of cosmic ray acceleration.

\section{FERMI LAT DATA}

The Fermi LAT is a gamma-ray telescope that detects photons in the energy range between $20 \mathrm{MeV}$ and $>500 \mathrm{MeV}$. It has a converter/tracker for direction measurement, a calorimeter for energy measurement and an anti-coincidence detector for charged background identification. The analysis presented here used data collected from the beginning of the mission (August 2008) to January 2017 and were analyzed with the recently improved effective area, point-spread function and energy resolution (Pass 8). Version v10r0p5 of the ScienceTools and instrument response functions with the event class SOURCE were used. Events were selected having a maximum zenith angle of $90^{\circ}$ and when the data quality was good. Data taken prior to 2008 October 27 have been excluded as they were affected by a 
higher level of background contamination, which is particularly important at the highest energies.

Events with energies from $5 \mathrm{GeV}$ to $500 \mathrm{GeV}$ were chosen within a $20^{\circ} \times 20^{\circ}$ region of interest around the position $\mathrm{RA}=233.5^{\circ}$, Dec $=-57^{\circ}$ (J2000). The LAT tools fit a model to the data including the residual charged particles and diffuse gamma-rays. A maximum likelihood technique (Mattox et al. 1996) was used to obtain the relevant source parameters and significance. The Galactic diffuse model used is given by the file gll_iem_v06.fits and the residual background and extragalactic emission were modeled with an isotropic component given by the file iso_P8R2_SOURCE_V6_v06.txt, which are provided by the LAT team. The data are binned spatially in counts maps using a scale of $0.05^{\circ}$ per pixel. For exposure calculation, ten logarithmically spaced bins per decade in energy were used. The sources included in the LAT Third Source Catalog (3FGL, Acero et al. 2015b) within the region of interest are considered in the fit, but only the normalizations of the sources located within $5^{\circ}$ around the center of the region were refitted with the increased data set, keeping their spectral shape fixed to the values reported in the catalog. The sources which are not significantly detected above $5 \mathrm{GeV}$ were removed from the model. The detection significance was estimated as the square root of the test statistic (TS), defined as $-2 \times \log \left(L_{0} / L\right)$, with $L_{0}$ and $L$ the likelihood values without and with the corresponding source, respectively.

After subtracting the best-fit model to the data, residuals are seen at the location of the radio shell of G323.7-1.0. Additional residuals of extended emission, unrelated to this SNR, are seen around the positions $\mathrm{RA}=238.46^{\circ}, \mathrm{Dec}=-53.4^{\circ}$ and $\mathrm{RA}=238.2^{\circ}$, Dec $=-56.2^{\circ}(\mathrm{J} 2000)$. This emission was accounted for with uniform disc templates of radii $0.5^{\circ}$ and $0.2^{\circ}$, respectively. The discs correspond to the sources $3 \mathrm{FHL}$ J1553.8-5325e and 3FHL J1552.7-5611e from the recently released Third Catalog of Hard Fermi-LAT Sources (The Fermi-LAT Collaboration 2017). The residuals map in the region of the SNR is shown in Fig. 1. The apparent residual emission extending to higher Galactic latitudes away from the SNR is not significant as demonstrated by fitting a point source at different locations near the region which results in a maximum TS of $\sim 2$.

\subsection{Source morphology}

Two spatial hypothesis to account for the emission were used for comparison, a uniform disc and ellipse. A spectral assumption of a simple power law $\left(\frac{d N}{d E} \propto E^{-\Gamma}\right)$ for the emission was used for both spatial templates.
For the uniform disc, a systematic search for the best fit template was carried out by moving the disc center along a $1^{\circ} \times 1^{\circ}$ grid centered near the geometrical center of the radio SNR, in steps of $0.1^{\circ}$, and changing its radius by $0.1^{\circ}$ in each step, from $0.1^{\circ}$ to $0.6^{\circ}$, and finding the maximum likelihood each time with the normalization and spectral index free in the fit. This results in five degrees of freedom in the fit. The uniform ellipse had a fixed shape tracing the radio border of the SNR, and thus the likelihood model with this morphology only had two degrees of freedom with respect to the null hypothesis, the normalization and spectral index.

The maximum TS value found for the disc hypothesis was 57.4 with a corresponding disc radius of $0.4_{-0.1}^{+0.2 \circ}$. The center of the best-fit template is located at the coordinates $\mathrm{RA}=233.5^{\circ}$, Dec $=-57.2^{\circ}$. The disc border is also shown in Fig. 1. The TS value found for the ellipse was 50 . For the rest of the analysis, the disc template was adopted in this work and systematic uncertainties in the spectral parameters were estimated to account for the choice of morphology.

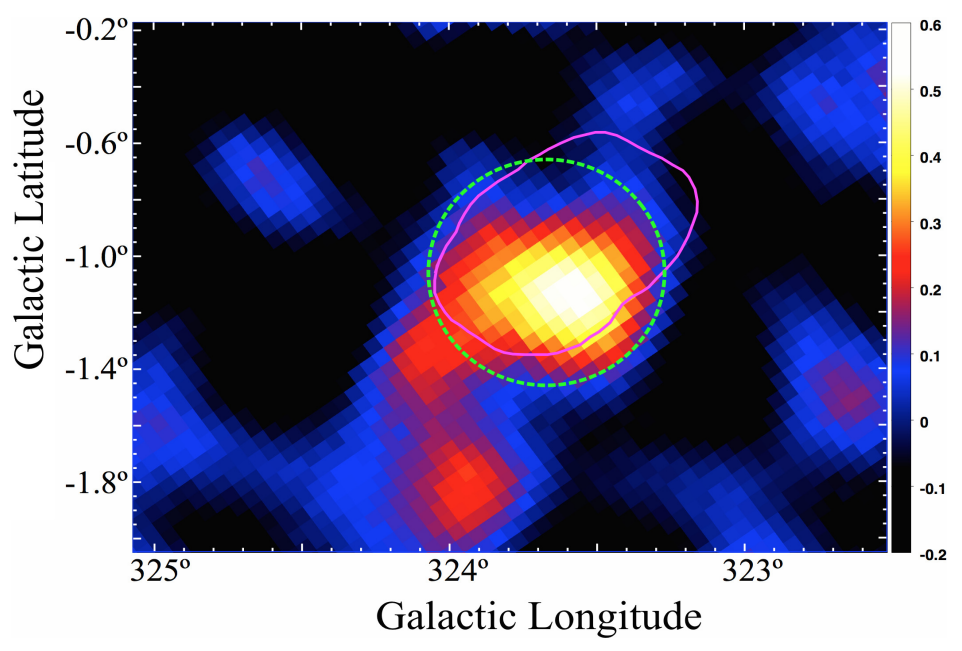

Figure 1. Map of residual emission above $5 \mathrm{GeV}$ in the region of the SNR G323.7-1.0 obtained after subtracting the best-fit model from the data. The map has units of counts per pixel and has been smoothed by a Gaussian with $\sigma=$ $0.15^{\circ}$. The dashed circle represents the best-fit disc template found in Section 2 and the solid line shows the approximate radio border of the SNR, taken from Green et al. (2014). No other sources from the LAT 3FGL catalog exist in this region.

\subsection{Spectral shape}

When fitting the spectrum to a power-law with an exponential cutoff the resulting spectral index and the TS value are the same as that from a simple power-law fit but the resulting cutoff energy is not constrained, as 
the fit gives $1.0 \pm 2.0 \mathrm{TeV}$. Therefore the simple powerlaw shape is used as the best-fit spectral shape in the $5-500 \mathrm{GeV}$ energy range.

The resulting spectral index and flux above $5 \mathrm{GeV}$ obtained were then $1.34 \pm 0.17_{\text {stat }} \pm 0.1_{\text {sys }}$ and $\left(2.2 \pm 0.6_{\text {stat }} \pm\right.$ $\left.0.5_{\text {sys }}\right) \times 10^{-10}$ photons $\mathrm{cm}^{-2} \mathrm{~s}^{-1}$. The systematic errors are estimated by combining the individual systematic errors resulting from uncertainty in the Galactic diffuse emission model and the source morphology. For the first one the normalization of the diffuse component was changed by $\pm 6 \%$ to estimate new spectral parameters of the source as typically done for analysis of LAT data (e.g., Abdo et al. 2009).

In order to probe the spectral behaviour in more detail the energy range was divided in five intervals and a likelihood fit was performed in each by keeping the values of the spectral parameters for all sources fixed to the ones obtained in the entire energy range, except for the normalization of the template representing HESS J1534-571. A value for the spectral energy distribution (SED) was estimated in each interval and a 95\%-confidence level upper limit was calculated for one of the intervals where the source TS is less than 7. The individual points are consistent with the fit for the whole data set, which supports the simple power-law spectral assumption. The data points and best-fit spectrum are shown in Fig. 2 together with the preliminary H.E.S.S. spectral points taken from Gottschall et al. (2016).

Finally, it is worth addressing the absence of a LAT source at the location of G323.7-1.0 in the recently released catalog of extended sources by The Fermi LAT Collaboration et al. (2017). Using the same data set as in their paper and the same cuts, a TS map was computed above $10 \mathrm{GeV}$ in the region for a point source hypothesis. The map showed a maximum value of 20 located inside the SNR shell, which passes the threshold of 16 used in the paper to search for extended emission with a uniform disc hypothesis. Adding a disc at this location only increases the TS by 10 , which is below the required value of 16 in their paper to claim a new extended source. The additional $\sim 40 \%$ integration time compared to the analysis by The Fermi LAT Collaboration et al. (2017) and the lower energy threshold used here can easily explain the new detection.

\section{DISCUSSION}

Extended $\mathrm{GeV}$ emission from a source compatible with the size of the SNR G323.7-1.0 was found with a significance of $7.5 \sigma$ above $5 \mathrm{GeV}$ in Fermi LAT data. This source is likely the counterpart of the $\mathrm{TeV}$ shell HESS J1534-571. Just as is seen for other TeV shells, the GeV spectrum of this source is hard.

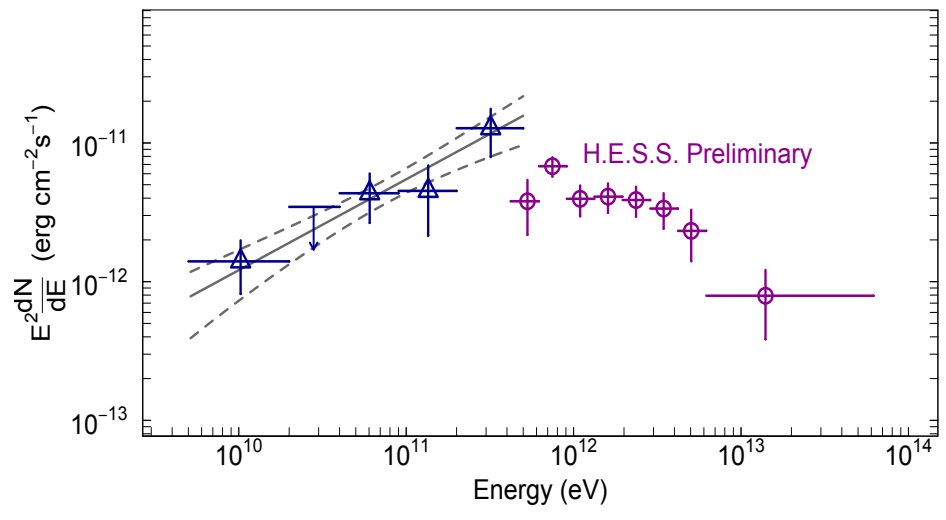

Figure 2. Gamma-ray SED of HESS J1534-571. The solid line shows the best-fit spectrum in the $5-500 \mathrm{GeV}$ energy range and the corresponding $1 \sigma$ statistical uncertainty is represented by the dashed lines. The LAT (triangles) and preliminary H.E.S.S. data points (circles) are also shown.

Using a recent parametrization of gamma-ray production cross sections for proton-proton interactions (Kafexhiu et al. 2014), the GeV-TeV data can be explained in a hadronic scenario with a particle distribution that is a power law with an exponential cutoff of the form $\propto \epsilon_{p}^{-s} \cdot e^{-\epsilon_{p} / \epsilon_{c}}$, where $\epsilon_{p}$ is the hadron energy and the required spectral index and cutoff energy are $s=1.5$ and $\epsilon_{c}=15 \mathrm{TeV}$. The total energy in the cosmic rays is $W_{p}=\left(1.07 \times 10^{49} \mathrm{erg}\right) \cdot d_{1}^{2} / n_{1}$, where $d_{1}$ and $n_{1}$ are the source distance to Earth and average density for the target material, in units of $1 \mathrm{kpc}$ and $1 \mathrm{~cm}^{-3}$, respectively. However, such a hard particle distribution is not consistent with predictions from standard cosmic ray acceleration theory. Some authors believe that, under very specific conditions in the ambient, hard gamma-ray emission can result from hadronic interactions of accelerated cosmic rays with dense, cold gas which does not emit thermal X-rays, but this scenario is not explored here. The reader is referred to the literature for a discussion about some of the corresponding models (e.g., Gabici \& Aharonian 2014, and references therein).

The hard GeV spectrum, on the other hand, can be naturally explained by leptonic emission. Recent analysis of LAT data from a TeV shell with a $\mathrm{GeV}$ spectrum similar to that of G323.7-1.0, showing a photon index of 1.4 (RCW 86), has concluded that the emission is likely leptonic (Ajello et al. 2016). Next, using a simple one-zone leptonic model with a particle distribution described by a power law with an exponential cutoff, a set of parameters consistent with the observations is derived. 
Fig. 3 shows the SED of HESS J1534-571 with the $\mathrm{GeV}$ spectrum obtained here, the preliminary H.E.S.S. data points (Gottschall et al. 2016) and the lower limit on the radio flux at a frequency of $843 \mathrm{MHz}$ from the Molonglo Galactic Plane Survey (Green et al. 2014). The figure also shows the leptonic emission calculated with the naima modeling package (Zabalza 2015) for a particle cutoff energy of $7 \mathrm{TeV}$ and a spectral index of $s=1.9$. The average source magnetic field used is $6 \mu \mathrm{G}$. Different contributions to the gamma-ray flux from IC scattering of far infrared (FIR) photon fields from heated dust and near-infrared star light (NIR), both described by modified blackbody spectra, as well as from the Cosmic Microwave Background (CMB), are included. The contribution from NIR is negligible for typical values in the Galaxy and the temperature and density found near the Solar System are used here, $3000 \mathrm{~K}$ and $0.4 \mathrm{eV} / \mathrm{cm}^{3}$ (e.g., Shibata et al. 2011; Vernetto \& Lipari 2016). With the adopted model parameters, a FIR temperature and density of $20 \mathrm{~K}$ and $0.8 \mathrm{eV} / \mathrm{cm}^{3}$ predict $\mathrm{GeV}-\mathrm{TeV}$ fluxes that are reasonably consistent with the data. This value of the FIR density is found at a distance of $5 \mathrm{kpc}$ from the Galactic center to the source using standard Galactic models (Shibata et al. 2011; Vernetto \& Lipari 2016). An estimation of the dust temperature using GALPROP (Grondin et al. 2011) for a Galactocentric distance of 5 kpc gives $\sim 30 \mathrm{~K}$, but we note that changing the dust temperature from $10 \mathrm{~K}$ to $50 \mathrm{~K}$ has little effect on the resulting IC fluxes. A Galactocentric distance of $5 \mathrm{kpc}$ would place the SNR at $\sim 4-5 \mathrm{kpc}$ from the Earth, near or at the Scutum-Centaurus arm of the Milky Way (e.g., Goodman et al. 2014). A value of $5 \mathrm{kpc}$ for the distance from the source to Earth requires a total leptonic energy in the source that is very reasonable, $\sim 0.15 \%$ of a typical supernova kinetic explosion energy of $10^{51} \mathrm{erg}$, and similar to the lepton energy content in other $\mathrm{TeV}$ shells such as RCW 86 (H. E. S. S. Collaboration et al. 2016).

Using these parameters, the model predicts a very low $2-10 \mathrm{keV}$ X-ray flux of $2.7 \times 10^{-14} \mathrm{erg} \mathrm{cm}^{-2} \mathrm{~s}^{-1}$ and a very steep X-ray $\operatorname{SED}\left(E^{2} \frac{d N}{d E} \propto E^{-3.1}\right)$.

The resulting nonthermal bremsstrahlung emission at gamma-ray energies is negligible for an average target density of $1 \mathrm{~cm}^{-3}$.

Although some model parameters could vary slightly and still account for the data reasonably well, the particle distribution is likely not much harder than the one proposed here. Although a model with a harder particle distribution $(s<1.9)$ better reproduces the observed $\mathrm{GeV}$ and $\mathrm{TeV}$ spectral shape, the radio flux lower limit becomes an important constraint against scenarios with harder spectra. Similarly, the particle index is likely not

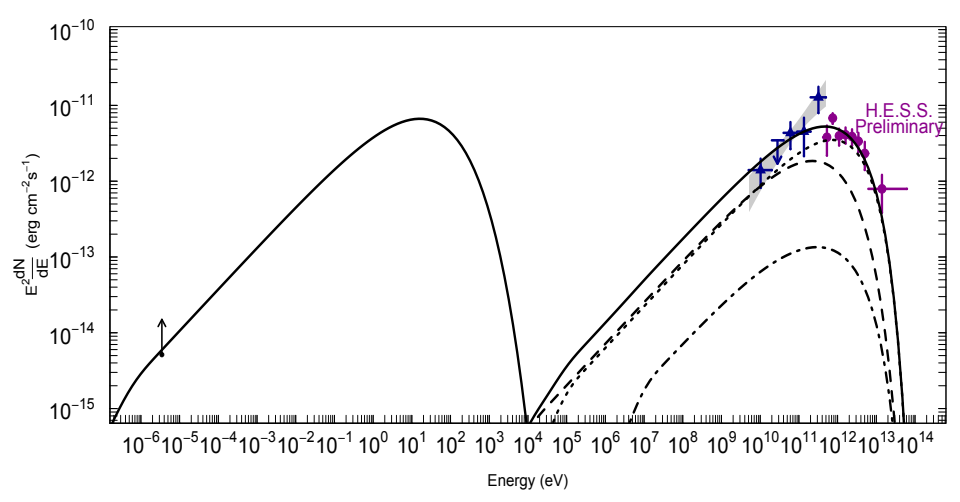

Figure 3. Leptonic model for the source HESS J1534-571 showing the two characteristic "bumps", one at low energies corresponding to synchrotron emission from high-energy electrons and another one resulting from IC scattering of ambient photons to gamma-ray energies by the same electrons. The components of IC emission are due to interactions with the CMB (dashed line), FIR (dotted line) and NIR photons (dash-dotted line). The Fermi LAT data from this work and the preliminary H.E.S.S. SED are shown. The lower limit on the flux from a $843 \mathrm{MHz}$ radio observation taken from Green et al. (2014) is also shown.

higher than $\sim 2.1$ because, first, that would require more energy in the electrons and, in order to keep the predicted X-ray fluxes from increasing significantly, a lower average magnetic field, which is already relatively low, but, most importantly, the shape of the predicted GeV$\mathrm{TeV}$ spectrum would deviate from the observations.

HESS J1534-571 could belong to a new class of relatively evolved SNR shells that are leptonic-dominated and show hard $\mathrm{GeV}$ spectra. Non-thermal X-rays are expected to decrease as a SNR evolves (Nakamura et al. 2012) and even though the evolution of the downstream magnetic field in a SNR is not well understood, it is expected to decrease with decreasing shock speeds (e.g., Völk et al. 2005). Other similar objects could include the relatively faint SNR G150.3+4.5 (Gao \& Han 2014; Ackermann et al. 2016). Follow up observations of HESS J1534-571 and modeling are necessary to confirm this scenario.

This work has received funding from the European Union's Horizon 2020 research and innovation programme under the Marie Skłodowska-Curie grant agreement No 690575. Partial financial support from Universidad de Costa Rica is also acknowledged. The comments made by the anonymous referee were very helpful to improve the quality of this paper. 


\section{REFERENCES}

Abdo, A. A., Ackermann, M., Ajello, M., et al. 2009, ApJL, 706, L1

Acero, F., Lemoine-Goumard, M., Renaud, M., et al. 2015a, A\&A, 580, A74

Acero, F., Ackermann, M., Ajello, M., et al. 2015b, ApJS, 218,23

—. 2016, ApJS, 224, 8

Ackermann, M., Ajello, M., Allafort, A., et al. 2013, Science, 339, 807

Ackermann, M., Ajello, M., Atwood, W. B., et al. 2016, The Astrophysical Journal Supplement Series, 222, 5. http://stacks.iop.org/0067-0049/222/i=1/a=5

Aharonian, F. A., \& Atoyan, A. M. 1996, A\&A, 309, 917

Ajello, M., Baldini, L., Barbiellini, G., et al. 2016, ApJ, 819,98

Araya, M., \& Cui, W. 2010, ApJ, 720, 20

Araya, M., \& Frutos, F. 2012, MNRAS, 425, 2810

Atwood, W. B., Abdo, A. A., Ackermann, M., et al. 2009, ApJ, 697, 1071

Bell, A. R. 1978, MNRAS, 182, 147

—. 2004, MNRAS, 353, 550

Capasso, M., Condon, B., Coffaro, M., et al. 2016, ArXiv e-prints, arXiv:1612.00258

Fukui, Y., Sano, H., Sato, J., et al. 2012, ApJ, 746, 82

Gabici, S., \& Aharonian, F. A. 2014, MNRAS, 445, L70

Gao, X. Y., \& Han, J. L. 2014, A\&A, 567, A59

Goodman, A. A., Alves, J., Beaumont, C. N., et al. 2014, ApJ, 797, 53

Gottschall, D., Capasso, M., Deil, C., et al. 2016, ArXiv e-prints, arXiv:1612.00261

Green, A. J., Reeves, S. N., \& Murphy, T. 2014, PASA, 31, e042

Grondin, M.-H., Funk, S., Lemoine-Goumard, M., et al. 2011, ApJ, 738, 42
H. E. S. S. Collaboration, Abramowski, A., Aharonian, F., et al. 2016, ArXiv e-prints, arXiv:1601.04461

Jogler, T., \& Funk, S. 2016, ApJ, 816, 100

Kafexhiu, E., Aharonian, F., Taylor, A. M., \& Vila, G. S. 2014, PhRvD, 90, 123014

Krymskii, G. F. 1977, Akademiia Nauk SSSR Doklady, 234, 1306

Mattox, J. R., Bertsch, D. L., Chiang, J., et al. 1996, ApJ, 461,396

Nakamura, R., Bamba, A., Dotani, T., et al. 2012, ApJ, 746,134

Pühlhofer, G., Brun, F., Capasso, M., et al. 2015, ArXiv e-prints, arXiv:1509.03872

Sakai, M., Yajima, Y., \& Matsumoto, H. 2011, PASJ, 63, S 879

Sano, H., Fukuda, T., Yoshiike, S., et al. 2015, ApJ, 799, 175

Shibata, T., Ishikawa, T., \& Sekiguchi, S. 2011, ApJ, 727, 38

Takeda, S., Bamba, A., Terada, Y., et al. 2016, PASJ, 68, S10

The Fermi-LAT Collaboration. 2017, ArXiv e-prints, arXiv:1702.00664

The Fermi LAT Collaboration, Ackermann, M., Ajello, M., et al. 2017, ArXiv e-prints, arXiv:1702.00476

Vernetto, S., \& Lipari, P. 2016, PhRvD, 94, 063009

Völk, H. J., Berezhko, E. G., \& Ksenofontov, L. T. 2005, A\&A, 433, 229

Zabalza, V. 2015, Proceedings of the 34th International Cosmic Ray Conference (ICRC2015), The Hague, The Netherlands, arXiv:1509.03319 